\title{
Preparation and Properties of Crosslinked Silk Fibroin Film
}

\author{
Ya-Fei WANG ${ }^{1,2}$, Chen LU ${ }^{1,2}$, Dan LONG ${ }^{1,2}$, Qiang ZHANG ${ }^{1,2}$, \\ Shu-Qin YAN ${ }^{1,2,}$, \\ ${ }^{1}$ School of Textile Science and Engineering, Wuhan Textile University, Wuhan 430073, \\ China \\ ${ }^{2}$ Advanced National and Local Joint Engineering Laboratory of Spinning, Weaving and \\ Clean Produce, Wuhan Textile University, Wuhan, 430073, \\ aemail: ysq_zq@163.com \\ ${ }^{*}$ Corresponding authors
}

Keywords: Silk fibroin, Film, Crosslinking, Structure, Properties.

\begin{abstract}
In this study, regenerated silk fibroin (SF) film was prepared by using 1-ethyl-3- (3-dimethylaminopropyl) carbodiimide hydrochloride (EDC) as cross-linking agent and N-hydroxysuccinimide (NHS), 2-morpholinoethanesulfonic acid (MES) as assistant agent. To evaluate the effects of EDC cross-linking agent on SF film performence, the water resistance, condensed structure, tensile strength, elongation, Young's modulus of crosslinked film were examined and analyzed. The water solubility and Young's modulus of the film with $20 \mathrm{wt} \%$ EDC decreased remarkably, while film elongation increased. Further, the FTIR, Raman spectra and $\mathrm{X}$-Ray Diffraction (XRD) were employed to investigate the molecule conformation transition and insight the mechanism of the crosslinking. The results demonstrated that crosslinked film contained more silk I when $\sim 20 \mathrm{wt} \%$ EDC (weight ratio to SF) was added. When more than $20 \mathrm{wt} \%$, the content of silk I decreased remarkably while that of silk II increased in the SF film. Due to the reaction of EDC, carboxylic acid groups and amino groups were bound by amide bond formation between the SF molecules. The NHS plays an activation of -OH group; the MES creates a faintly acid condition to be beneficial for the cross-link reaction. This study provided an important method to prepare insoluble SF materials and promoted application of the SF materials.
\end{abstract}

\section{Introduction}

Silk fibroin (SF), the natural protein, is all known as texture materials. In recent years, its attractive advantages in biocompatibility and biomedical applications have been motivated more research on development of SF based biomaterials [1]. There are many studies about the SF materials applied in controlled drug delivery system, blood vessel engineering, peripheral nerve regeneration materials, femur defects, bone tissue engineering [2].

The SF film can be prepared by casting the SF solution followed by air dried. However, it was limited in practical application due to the weak intermolecular force and solubility. To overcome the drawbacks, many researchers have been concentrating on preparing insoluble SF films. The influence of various organic solvent treatments on the non-crystallize domains in the SF film was studied [3]. The results showed that ethanol treatment would induce condense structure of SF film transformed to silk II. The same results were obtained that the structures of SF films were changed from random coil to silk II by water-methanol solutions (60 80vol \% methanol concentration) treatments $[4,5]$. Above-mentioned methods could obtain the insoluble 
SF films, however, these treatments induced the structure transformation to $\beta$-sheet, and the films were generally rigid and brittle. To further improve the insolubility and flexibility of SF film, the more crosslinking agents were examined. The low polyhydric alcohol was employed to crosslink with fibroin to achieve the goal of insoluble SF film, which can improve the mechanical properties of the SF film such as strength, elongation percentage, water vapor permeability in increase, while water permeability remarkably in decrease [6].

Although water resistance of the SF film could be improved by the above-mentioned methods, there are still some limitations. 1-ethyl-3- (3-dimethylaminopropyl) carbodiimide hydrochloride (EDC) was chosen as crosslinking agent over past few years [7-10]. It showed effective crosslinking reaction took place between collagen and EDC, and this material is biocompatible, mechanically superior.Thus, to improve the water resistance, mechanical properties and biocompatibility of the film, (3-dimethylaminopropyl) carbodiimide hydrochloride (EDC) was selected as crosslinking agent. N-hydroxy succinimide (NHS), 2-morpholinoethanesulfonic acid (MES) were used as assistant agents. We also evaluated the influence of the degree of crosslinking on structure, water resistance and mechanical properties of crosslinked film.

\section{Experimental}

\section{Preparation of Regenerated $B$. mori SF film}

Raw silk fibers, purchased from Zhejiang province of China, were degummed three times with $0.05 \%$ (w/w) $\mathrm{Na}_{2} \mathrm{CO}_{3}$ solution at $98^{\circ} \mathrm{C}$ for $30 \mathrm{~min}$ and rinsed thoroughly and dried in oven at $60^{\circ} \mathrm{C}$. The extracted SF was dissolved in a ternary solvent system of $\mathrm{CaCl}_{2} / \mathrm{CH}_{3} \mathrm{CH}_{2} \mathrm{OH} / \mathrm{H}_{2} \mathrm{O}(1: 2: 8$ in molar ration $)$ at $70 \pm 2^{\circ} \mathrm{C}$ for $1 \mathrm{~h}$. SF solution with 3.0 wt $\%$ concentration, which measured by weighting method, was obtained after dialysis. The SF solution was mixed with different degree (percent of the quality to SF in the solution) of crosslinking agents (EDC, NHS and MES, Sigma Chemicals, Shanghai). The SF films with thickness about $50 \mu \mathrm{m}$ were prepared by casting the SF solutions on the polyethylene dish and dried at $60^{\circ} \mathrm{C}$.

To evaluate the crosslinking effect of the contents of EDC, NHS and MES on the SF films, three different experiment groups were designed. Group one, changed the content of EDC (EDC/ NHS=2:1, no MES), the content of EDC is $10 \%, 15 \%, 20 \%$, $25 \%, 30 \%$ respectively. Group two, changed the content of NHS (remain the content of EDC 20\% unchanged, no MES), the finally content of EDC and NHS were as follows: EDC20\%, NHS 0\%; EDC 20\%, NHS 6.67\% (3:1); EDC 20\%, NHS 8\% (2.5:1); EDC $20 \%$, NHS $10 \%$ (2:1); EDC 20\%, NHS 13.3\% (1.5:1); EDC 20\%, NHS 20\% (1:1). The group three, changed the content of MES (remain the content of EDC 20\% and NHS $10 \%$ unchanged), the weight ratio of MES was $0 \%, 5 \%, 10 \%, 15 \%, 20 \%, 25 \%$.

\section{Water Solubility and Mechanical Properties}

Water solubility of SF film was determined by shaking of $250 \mathrm{mg}$ of film and in $25 \mathrm{ml}$ of water at $37^{\circ} \mathrm{C}$ for $24 \mathrm{~h}$. Since direct weighing of the remaining solid was cumbersome, the weight of solubilized fraction was determined by eq. (1), using ultraviolet absorbance of the supernatant at $278 \mathrm{~nm}$.

$$
D=\frac{K V A}{G} \times 100 \%
$$


Where $\mathrm{D}$ (wt.\%) is water solubility, $\mathrm{K}$ is $0.1832 \mathrm{mg} / \mathrm{ml}$, experimental constant determined from weight loss, A is absorbance at $278 \mathrm{~nm}, \mathrm{~V}(\mathrm{ml})$ is the volume of the solution, $\mathrm{G}(\mathrm{mg})$ is the initial weight of sample.

Tensile strength and elongation at break were measured by an Instron 33655 Tester at $20^{\circ} \mathrm{C}, 65 \% \mathrm{RH}$ with a head speed of $20 \mathrm{~mm} / \mathrm{min}$. The specimen was $30 \mathrm{~mm}$ wide and 30 mm long between clamps.

Initial tensile modulus was performed with an Y391 Yarn Elasticity Tester. The samples were cut into $120 \times 5 \mathrm{~mm}$ strips. The elongation value $(\Delta \mathrm{L})$ was obtained after a drawing load $(\mathrm{P})$ of $0.735 \mathrm{~N}$ was exerted on the samples for $5 \mathrm{~s}$. The measurement was performed under the following conditions: pretension of $0.2 \mathrm{~N}$ and gauge length $\left(\mathrm{L}_{0}\right)$ of $50 \mathrm{~mm}$. The initial tensile modulus was calculated as follows:

$$
E=\frac{P \times L_{0}}{S \times \Delta L}
$$

Where $E\left(\mathrm{~N} / \mathrm{mm}^{2}\right)=$ initial tensile modulus, $\mathrm{P}(\mathrm{N})=$ drawing load, $\mathrm{L}_{0}(\mathrm{~mm})=$ specimen gripping length at pretension, $\mathrm{S}\left(\mathrm{mm}^{2}\right)=$ specimen cross-section area, $\Delta \mathrm{L}$ $(\mathrm{mm})=$ elongation value in $5 \mathrm{~s}$.

\section{Structure Analysis of the Crosslinked Film}

X-Ray Diffraction (XRD). X-ray diffraction was performed by X'Pert-Pro MPD diffractometer, and $\mathrm{CuK} \alpha$ radiation with a wavelength of $1.5406 \AA$ was used. The scanning speed was $2^{\circ} / \mathrm{mm}$. The diffraction intensity curves with $2 \theta$ from 5 to $45^{\circ}$ were obtained.

Fourier Transform Infrared (FT-IR). The films were cut into micro-particles with radius less than $40 \mu \mathrm{m}$, and then samples were prepared in $\mathrm{KBr}$ pellets. FT-IR spectra were obtained with a Nicolet Avatar-IR360.

Raman spectra. Raman spectra were recorded using a Dilor LabRam-1B spectrometer, operating at a resolution of $1 \mathrm{~cm}-1$. The Spectra Physics Model 164 argon ion laser was operated at $632.8 \mathrm{~nm}$ with about $6 \mathrm{~mW}$ of power.

\section{Results and Discussions}

\section{Water Solubility of the Film}

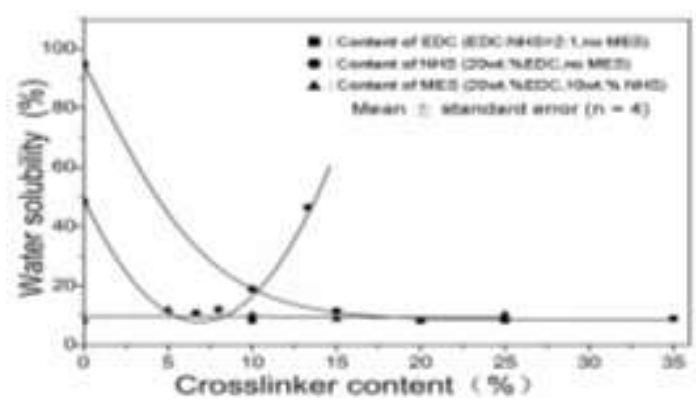

Figure 1. Water solubility of the SF film added crosslinking agents with different ratio of SF in the solution

As shown in Fig. 1, the water resistance was improved remarkably after cross-linked with EDC and NHS (EDC:NHS=2:1, no MES). The effective crosslinking reaction took place between SF molecules, and was enhanced with an increase of the crosslinking agent content. And it reached the optimal crosslinking effect when the 
$20 \%$ EDC was used. The water solubility was comparatively low, with 5-10 wt.\% NHS (remain the content of EDC 20\% unchanged, no MES) addition. With more than 10 wt.\% NHS addition, the water solubility became corresponding increase. These results demonstrated that the effect of crosslinking for improving mechanical properties is apparently greatest at the ratio of EDC: NHS of $1 / 4$ to $1 / 2$. No significant effect of adding MES has been observed.

\section{Young's Modulus, the Strength and Elongation of the Film}

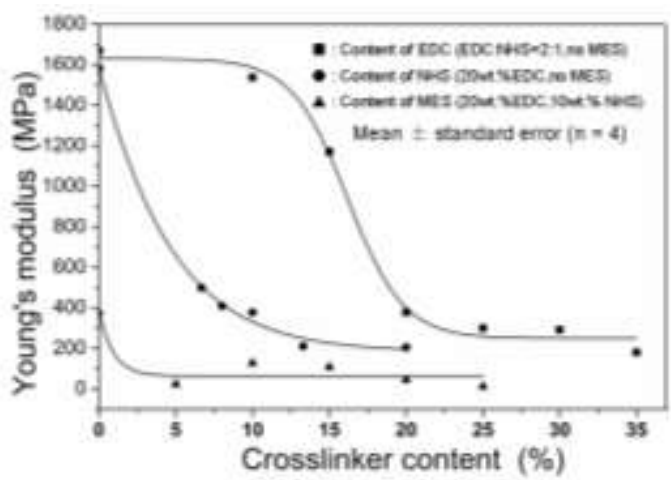

Figure 2. Young's modulus of the SF film added crosslinking agents with different ratio of SF in the solution

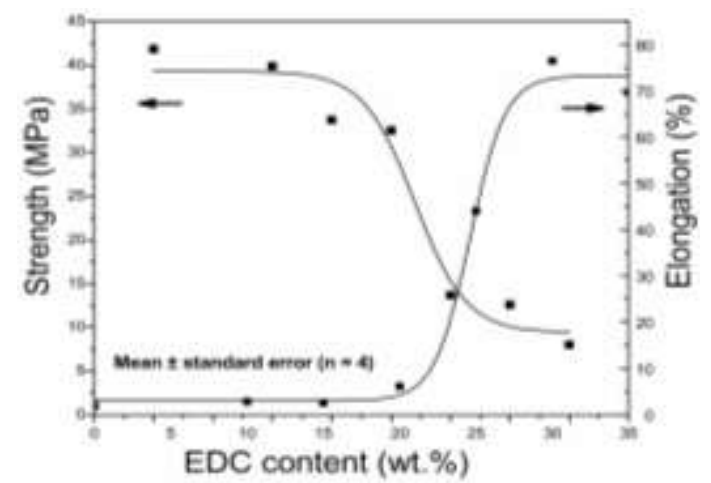

Figure 3. Effect of EDC content (EDC: $\mathrm{NHS}=2:$, no MES )on strength and elongation of the SF film added crosslinking agents with different ratio of SF in the solution.

Young's modulus is an important indicator to measure the flexibility of materials. As shown in Fig. 2, the Young's modulus of the films decreased gradually with increasing of the content of EDC and NHS, indicating that the flexibility of the films improved gradually. The Young's modulus of the crosslinked films was smaller when added 20 wt.\% EDC. While more than $20 \mathrm{wt} . \%$ the Young's modulus has no obvious decrease. Young's modulus decreases remarkably when added $13.3 \mathrm{wt} \%$ NHS and $5 \mathrm{wt} . \%$ MES. However, with an increase of the NHS and MES, it has no further effective. As shown in Fig. 3, the tensile elongation at break of the silk film increased while tensile strength at break decreased with an increase of EDC and NHS content (EDC:NHS=2:1). Especially, the range of the increase and decrease is wide with more than $20 \mathrm{wt} . \%$ DEC addition.

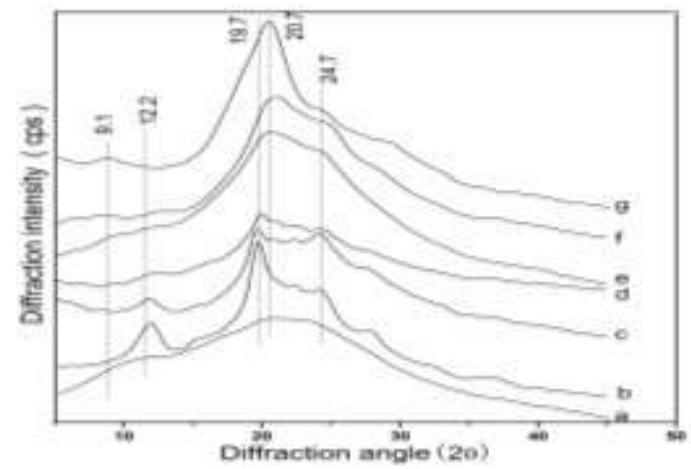

Figure 4. XRD curves of the different SF materials. (a) SF film without crosslinker, (b) $10 \mathrm{wt} . \%$ EDC and 5 wt.\% NHS, (c) 20 wt.\% EDC and 10 wt.\% NHS, (d) 35 wt.\% EDC and 17.5 wt.\% NHS, (e) 20 wt.\% EDC and 10 wt.\% NHS and 10 wt.\% MES, (f) 20 wt.\% EDC and 10 wt.\% NHS and 20 wt.\% MES, (g) SF fibers without crosslinker. 


\section{X-ray Diffraction}

XRD studies on $\mathrm{B}$. mori $\mathrm{SF}$ using $\mathrm{CuK} \alpha$ radiation indicated the main diffraction peaks of Silk I crystal at $12.2^{\circ}\left(\mathrm{d}=7.25 \AA\right.$, medium strong), $19.7^{\circ}$ (d=4.5 $\AA$, strong), $24.7^{\circ}$ $(\mathrm{d}=3.60 \AA$, medium $)$, and $28.2^{\circ}(\mathrm{d}=3.16 \AA$, medium $)$, while those of silk II are at $9.1^{\circ}$ (d=9.7 $\AA$, medium strong), $18.9^{\circ}\left(\mathrm{d}=4.69 \AA\right.$, medium strong), $20.7^{\circ}(\mathrm{d}=4.30 \AA$, very strong), and $24.3^{\circ}(\mathrm{d}=3.66 \AA$, medium strong) [10, 11]. Fig. 4 shows the X-ray diffraction curves of the SF films with different degree of crosslinking agents. The uncross-linked film (Fig. 4a) shows weak peaks at around $12.2^{\circ}$ and $19.7^{\circ}$, indicating the coexistence of silk I and random coil. The silk fiber (Fig. $4 \mathrm{~g}$ ) shows strong peaks at around $9.1^{\circ}$ and $20.7^{\circ}$, which attribute to the silk II structure. The SF films cross-linked with 10wt.\% EDC and 5wt.\% NHS (Fig. 4b), 20 wt.\% EDC and 10 wt.\% NHS (Fig. 4c) show intense strong peak at around $19.7^{\circ}$, medium strong peak at around $24.7^{\circ}$ and $12.2^{\circ}$, indicating the presence of significant amount of silk I. The curves of SF films cross-linked with more than 20\% EDC (Fig. 4d) or added the MES assistant crosslinking agent (Fig. 4 (e, f)) show that the smooth peaks at $20.7^{\circ}$ and $9.1^{\circ}$ gradually appeared, and at $19.7^{\circ}$ displayed a contrary tendency, the peaks at around $12.2^{\circ}$ and $24.7^{\circ}$ become weaker, which indicating the decreases in silk I and increases in silk II with the increase of EDC and NHS content or added MES.

\section{FT-IR Spectra}

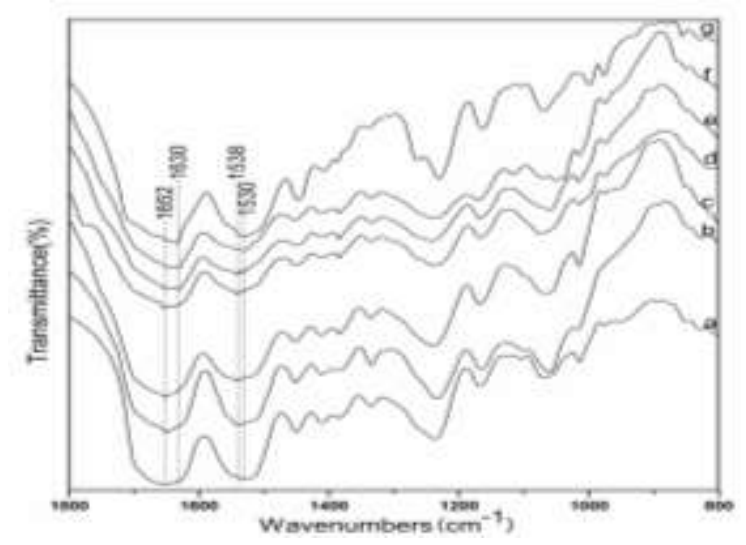

Figure 5. FT-IR spectra of the different SF materials. (a) SF film without crosslinker, (b) 10 wt.\% EDC and 5 wt.\% NHS, (c) 20 wt.\% EDC and 10 wt.\% NHS, (d) 35 wt.\% EDC and 17.5 wt.\% NHS, (e) 20 wt.\% EDC and 10 wt.\% NHS and 10 wt.\% MES, (f) 20 wt.\% EDC and 10 wt.\% NHS and 20 wt.\% MES, (g) SF fibers without crosslinker.

Previous studies on the molecular conformation of SF revealed that $\alpha$-form usually exhibits characteristic absorption bands at 1650-1655 $\mathrm{cm}^{-1}$ (amide I), 1525-1540 cm (amide II), $1266 \mathrm{~cm}^{-1}$ (amide III), and $669 \mathrm{~cm}^{-1}$ (amide V), $\beta$-sheets have characteristic peaks at 1620-1635 $\mathrm{cm}^{-1}$ (amide I), $1530 \mathrm{~cm}^{-1}$ (amide II), 1230-1235 $\mathrm{cm}^{-1}$ (amide III), and $700 \mathrm{~cm}^{-1}$ (amide V), and absorbance peaks at $1655-1660 \mathrm{~cm}^{-1}$ (amide I), 1535-1545 $\mathrm{cm}^{-1}$ (amide II), $1235 \mathrm{~cm}^{-1}$ (amide III), and $650 \mathrm{~cm}^{-1}$ (amide V) were assigned to the random coil conformation $[10,11]$. The spectrum of uncross-linked SF films (Fig. 5a) shows absorption bands around $1654 \mathrm{~cm}^{-1}$ and $1538 \mathrm{~cm}^{-1}$, indicating the presence of silk I and random coil conformation. The spectrum of the silk fibers (Fig. 5g) shows strong absorption bands around $1630 \mathrm{~cm}^{-1}$ and $1530 \mathrm{~cm}^{-1}$, which mainly attributed to the silk II structure. The spectrum of SF films cross-linked with $10 \mathrm{wt} . \%$ EDC and 5 wt.\% NHS (Fig. 5b), 20 wt.\% EDC and 10 wt.\% NHS (Fig. 5c) showed that the bands are mainly at around $1652 \mathrm{~cm}^{-1}$ and $1538 \mathrm{~cm}^{-1}$, which attributed to silk I. Meanwhile, the spectrum of SF films cross-linked with more than 20\% EDC (Fig. 5d) or added the 
MES assistant crosslinking agent (Fig. 5 (e, f)) show that besides at around $1652 \mathrm{~cm}^{-1}$ and $1538 \mathrm{~cm}^{-1}$, the new absorption bands at around $1630 \mathrm{~cm}^{-1}$ and $1530 \mathrm{~cm}^{-1}$ appeared, which indicated that part of the condensed structure changed from silk I to silk II.

\section{Raman Spectra}

Previous studies on the molecular conformation of SF revealed that Raman spectra of SF films exhibit strong bands for silk I at around $1660 \mathrm{~cm}^{-1}$ (amide I), $1252 \mathrm{~cm}^{-1}$ (amide III) and $1270 \mathrm{~cm}^{-1}$ (amide III), and for silk II at around $1676 \mathrm{~cm}^{-1}$ (amide I) and 1233 $\mathrm{cm}^{-1}$ (amide III) [12].

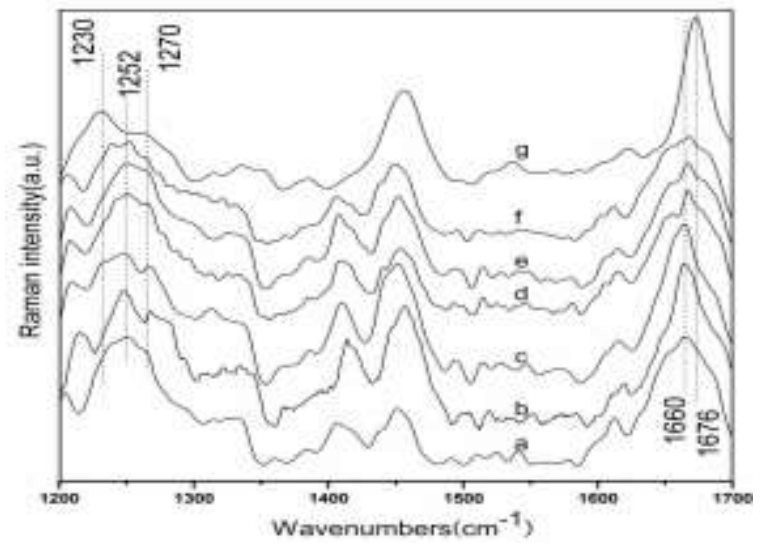

Figure 6. Raman spectra of the different SF materials. (a) SF film without crosslinker, (b) 10 wt.\% EDC and 5 wt.\% NHS, (c) 20 wt.\% EDC and 10 wt.\% NHS, (d) 35 wt.\% EDC and 17.5 wt.\% NHS, (e) 20 wt.\% EDC and 10 wt.\% NHS and 10 wt.\% MES, (f) 20 wt.\% EDC and 10 wt.\% NHS and 20 wt.\% MES, (g) SF fibers without crosslinker.

The Raman spectra of uncross-linked SF film (Fig. 6a) exhibits smooth bands near 1660 (amide I), 1252 (amide III), which are the characteristic bands of silk I structure. The silk fiber (Fig. $6 \mathrm{~g}$ ) shows amide I bands at $1676 \mathrm{~cm}^{-1}$ and amide III bands at 1230 $\mathrm{cm}^{-1}$, all these bands attribute to silk II structure. The 10wt.\% EDC and $5 \mathrm{wt} \%$ NHS cross-linked film (Fig. 6b), 20 wt.\% EDC and 10 wt.\% NHS cross-linked film (Fig. 6c) show that the amide I bands around $1660 \mathrm{~cm}^{-1}$, amide III bands at 1270 and $1252 \mathrm{~cm}^{-1}$ are strengthened, these results indicate that the molecular conformation of the films are mainly silk I. However, the spectrum of SF films cross-linked with more than $20 \%$ EDC (Fig. 6d) or added the MES assistant crosslinking agent (Fig. 6 (e, f)) show that the amide I band shifts to $1670 \mathrm{~cm}^{-1}$ from $1660 \mathrm{~cm}^{-1}$, amide III bands at 1270 and 1252 $\mathrm{cm}^{-1}$ are weakened, these results further confirm that the part of the condensed structure changed from silk I into silk II.

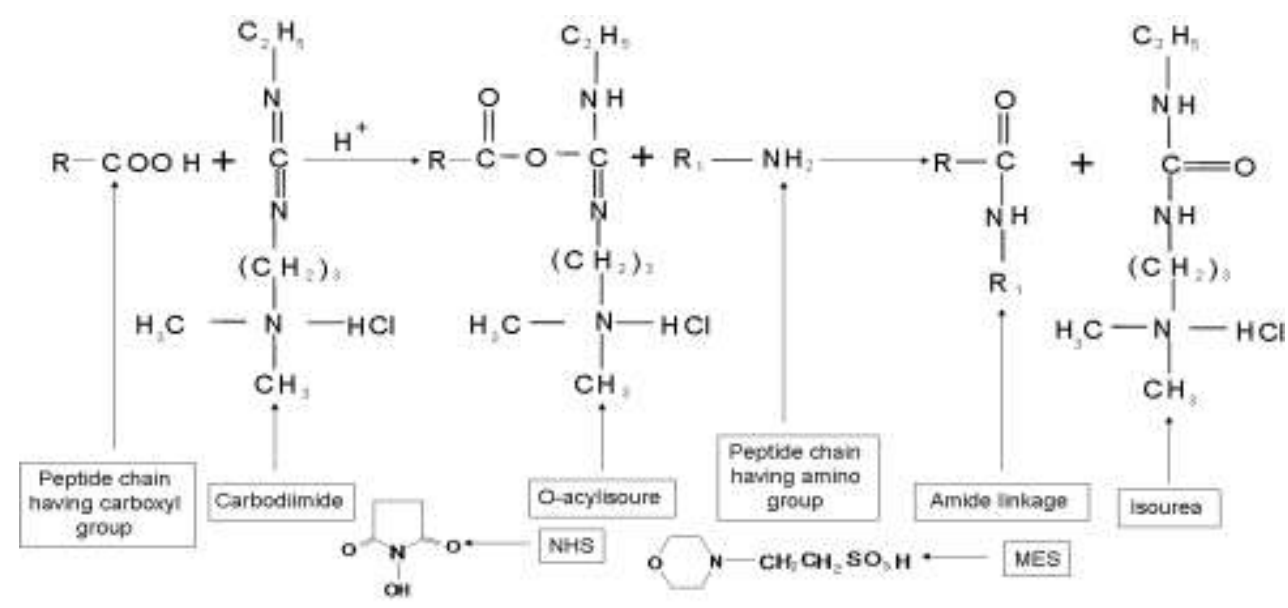

Figure 7. Reaction mechanisms of EDC with carboxyl and amino group in the SF 
As shown in Fig. 7, EDC/NHS crosslinking step which was applied with EDC in combination with NHS involves the activation of the residual carboxylic acid groups with EDC to give an O-acylisourea group. In this procedure, carboxylic acid groups are activated by EDC and NHS which subsequently react with adjacent free amine groups forming amide bonds. According to the chemistry structural formula of NHS and MES as shown in Fig. 7, in the presence of NHS and MES, the O-acylisourea group is converted to a NHS activated carboxylic acid group and a MES activated sulfonic acid group, which are highly reactive towards amine groups, and a crosslink is formed through formation of an amide bond. The $\mathrm{O}$-acylisourea groups formed upon reaction of a carboxylic acid group with EDC can rearrange via an $\mathrm{N}$-acrewfdscxzryl shift to a hydrolytically stable $\mathrm{N}$-acylurea group. MES was allowed to react with EDC or a mixture of EDC and NHS. The occurrence of the $\mathrm{N}$-acyl shift was related to differences in the number of free carboxylic acid groups present before and after treatment of MES with EDC or EDC and NHS.

\section{Summary}

Effective crosslinking reaction took place between fibroin and EDC in the regenerated SF film. Added NHS and MES could promote the reaction and improve the flexibleness of the film. The crosslinking reaction contribute to reduce silk I component and increase amorphous domains in crosslinked SF film. The film cast from regenerated SF solution containing about $20 \mathrm{wt} . \%$ EDC, $10 \mathrm{wt} . \%$ NHS and $5 \mathrm{wt} . \%$ MES showed not only high water resistively but also remarkable flexibleness. This approach provides a base data for SF materials preparation.

\section{Acknowledgement}

This work was supported by the National Nature Science Foundation of China (51303141, and 51403163), Educational Commission of Hubei Province, China (154004) and Nature Science Foundation of Wuhan Textile University (153095, 153043).

\section{References}

[1] Moy R, Lee A and Zalka A. Commonly used suture materials in skin surgery. Am Fam Physician 1991; 44: 2123-8.

[2] Zhang Q, Yan S and Li M. Silk Fibroin Based Porous Materials. Materials 2009; 2: 2276-95.

[3] Wang CH, Hsieh CY, Hwang J. Flexible Organic Thin-Film Transistors with Silk Fibroin as the Gate Dielectric. Adv Mater 2011; 23:1630-4.

[4] Taketani I, Nakayama S, Nagare S, Senna M, The secondary structure control of silk fibroin thin films by post treatment. Appl Surf Sci 2005; 244: 623-6.

[5] Tsukada M, Gotoh Y, Nagura M, Minoura N, Kasai N, Freddi G, Structural changes of silk fibrion membranes induced by immersion in methanol aqueous solution. $\mathrm{J}$ Polym Sci Pol Phys 1994; 32: 961-8.

[6] Jin HJ, Park J, Valluzzi R, Cebe P, Kaplan DL. Biomaterial Films of Bombyx Mori Silk Fibroin with Poly (ethylene oxide) Biomacromolecules 2004; 5: 711-7. 
[7] Zeeman R, Dijkstra PJ, van Wachem PB, van Luyn MJ, Hendriks M, Cahalan PT, Feijen J. Successive epoxy and carbodiimide cross-linking of dermal sheep collagen. Biomaterials 1999; 20: 921-31.

[8] Zhang Q, Zhao Y, Yan S, Yang Y, Zhao H, Li M, Lu S and Kaplan D. Preparation of uniaxial multichannel silk fibroin scaffolds for guiding primary neurons. Acta Biomater 2012; 8: 2628-38.

[9] Yan S, Li M, Zhang Q, Wang J. Blend films based on silk fibroin/hyaluronic acid. Fiber Polym 2013; 14: 188-94.

[10] Yan S, Zhang Q, Wang J, Liu Y, Lu S, Li M and Kaplan D. Silk fibroin/chondroitin sulfate/hyaluronic acid ternary scaffolds for dermal tissue reconstruction. Acta Biomater., 2013; 9: 6771-82.

[11] Li M, Ogiso M, Minoura N. Enzymatic degradation behavior of porous silk fibroin sheets. Biomaterials 2003; 24: 357-65.

[12] Zheng Z, Wei Y, Yan S, Li M. Preparation of Regenerated Antheraea yamamai Silk Fibroin Film and Controlled Molecular Conformation Changes by Aqueous Ethanol Treatment. J Appl Polym Sci 2010; 116: 461-7. 